\title{
Prägend wie kein anderer - zur Emeritierung unseres Mitherausgebers
}

Am 1. Dezember 2016 hat Mathias Berger nach 16 Jahren Direktorat der Klinik für Psychiatrie und Psychotherapie des Universitätsklinikums Freiburg die Amtsgeschäfte an seine Nachfolgerin Katharina Domschke übergeben. Damit wurde nicht nur einer unserer Mitherausgeber emeritiert, der InFo Neurologie \& Psychiatrie von Anfang an begleitet hat, sondern ein Kollege, der das Fach Psychiatrie und Psychotherapie wie kein anderer geprägt hat.

Nach dem Studium in Köln und der Promotion im Jahr 1974 arbeitete Mathias Berger zunächst von 1974 bis 1978 an der Universitätsnervenklinik Köln, um anschließend an das MaxPlanck-Institut für Psychiatrie in München zu wechseln, wo er seine psychiatrische Facharztzeit absolvierte und beide Facharztweiterbildungen (Facharzt für Neurologie und Psychiatrie, Zusatztitel Psychotherapie) abschloss. Nach der Habilitation im Jahr 1985 war er zunächst stellvertretender und dann Leiter der Abteilung Psychiatrie II. 1986 wurde Mathias Berger auf eine C3-Professur am Zentralinstitut für Seelische Gesundheit in Mannheim berufen, wo er bis 1990 leitender Oberarzt war. Von 1990 bis 2016 war er dann C4-Professor für Psychiatrie und Psychotherapie am Universitätsklinikum Freiburg.

Neben seinen wichtigen Forschungsbeiträgen im Bereich der Regulation der HPA-Achse und des Schlafes steht sein Name für die Einführung störungsorientierter, Schulen übergreifender und evidenzbasierter Psychotherapien. Mehrere dieser Konzepte aus der „Freiburger Schule“ sind inzwischen an vielen Kliniken in Deutschland etabliert und haben zu einer deutlichen Verbesserung der Versorgung psychisch kranker Menschen geführt. Mathias Berger war auch einer der ersten, der in Zusammenarbeit mit dem Deutschen Cochrane-Zentrum in Freiburg das Thema evidenzbasierte Medizin in der Psychiatrie und Psychotherapie voranbrachte. Dieses, an wissenschaftlichen Kriterien und nicht an Plausibilitäten orientierte Denken war für ihn

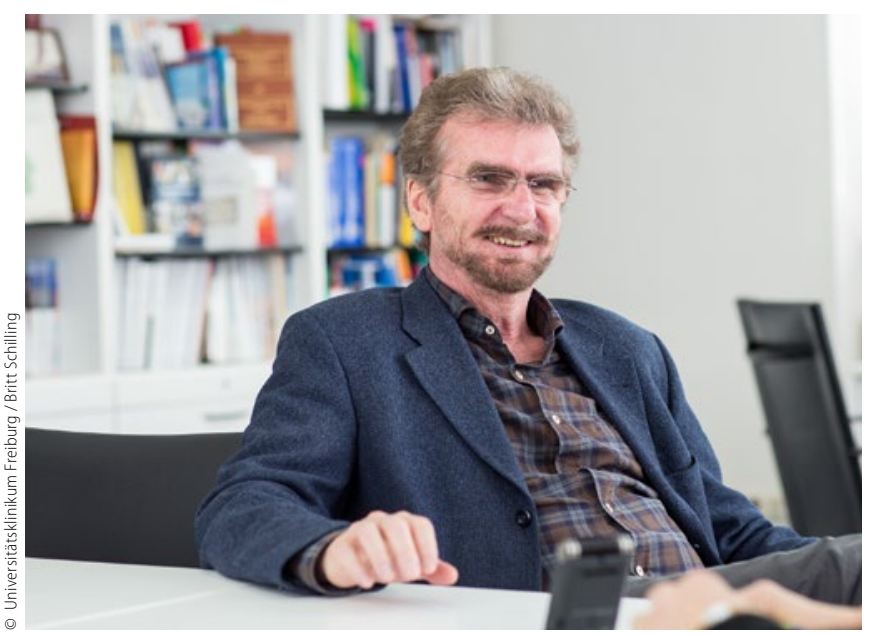

Blickt gelassen, aber mit weiterhin großer Leidenschaft für sein Fach in die Zukunft - Professor Dr. med. Mathias Berger.

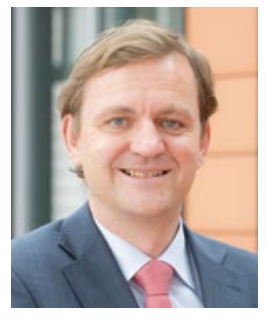

Prof. Dr. med. Klaus Lieb, Mainz

Direktor der Klinik für Psychiatrie und

Psychotherapie

Universitätsmedizin Mainz

Untere Zahlbacher Str. 8, 55131 Mainz

E-Mail: klaus.lieb@unimedizin-mainz.de

immer wegweisend und Anlass, InFo Neurologie \& Psychiatrie von Anfang an zu unterstützen. Gleichzeitig ist es eine der Grundlagen für sein Lehrbuch „Psychische Erkrankungen“, das in der fünften Auflage bereits ein Klassiker ist.

Es ist nicht möglich, eine so vielschichtige und erfolgreiche Hochschullehrer-Persönlichkeit in einem kurzen Editorial adäquat zu würdigen. Daher sei auf eine ausführliche Würdigung von Mathias Berger und einen vertieften Einblick in die „Freiburger Schule“ im kürzlich bei Elsevier erschienenen Buch „Psychiatrie und Psychotherapie 2.0“ verwiesen. Drei Initiativen sollen hier aber dennoch kurz genannt werden, die deutlich machen, wie erfolgreich sich Mathias Berger für die Interessen des Faches Psychiatrie und Psychotherapie eingesetzt hat: Erstens sein erfolgreiches Engagement, die Psychotherapieausbildung besser in der Psychiatrie zu verankern. Er war zusammen mit dem Kollegen Buchkremer ganz entscheidend an der Einführung eines neuen Facharztes für Psychiatrie und Psychotherapie auf dem Ärztetag 1992 beteiligt. Zweitens hatte Mathias Berger seine DGPPN-Präsidentschaft in den Jahren 2002 bis 2004 erfolgreich genutzt, eine deutschlandweite Analyse der psychiatrischen Versorgung in Gang zu setzen, die die Grundlagen für weitere Planungen schuf sowie den DGPPN-Kongress in Berlin fest zu etablieren und damit zum größten europäischen Psychiatrie-Kongress mit fast 10.000 Teilnehmern zu machen. Und drittens ist sein großes Engagement in der Abwehr des PEPPSystems im Jahr 2016 zu erwähnen. Ohne Mathias Berger wäre es wohl nicht gelungen, die - nicht nur für Universitätskliniken - existenzielle Bedrohung einer missglückten Systemreform abzuwenden.

Ich bin sicher, dass die Emeritierung von Mathias Berger nur eine formale Zäsur ist. Er wird sich für InFo Neurologie \& Psychiatrie und das Fach Psychiatrie und Psychotherapie weiter mit großer Leidenschaft engagieren und seinen Prinzipien treu bleiben. Der bekannte Schriftsteller Rainald Goetz, der Mathias Berger in den 1980er-Jahren in München kennengelernt hatte, bewies eine gute Nase, als er in seinem Buch „Irre“ (Suhrkamp 1986, Seite 117) schrieb: „Man kann eine außerordentliche Universitätskarriere machen, ohne sich zu verraten. Berger ist der Beweis." Schöner kann man es eigentlich nicht sagen! 